\title{
Comparison of three probability models for offshore structural response due to Morison wave loading
}

\author{
G. Najafian \\ Department of Engineering, The University of Liverpool, UK
}

\begin{abstract}
Offshore structures are exposed to random wave loading in the ocean environment and hence the probability distribution of their response to wave loading is of great value in probabilistic analysis of these structures. Due to nonlinearity of the wave load mechanism and also due to intermittency of wave loading on members in the splash zone, the response is often non-Gaussian. Two probability models have frequently been used in the past for offshore structural response due to random Morison wave loading: a) the Pierson--Holmes distribution and b) a third-order polynomial function of a Gaussian random variable. Recent work has, however, demonstrated that none of these two models can accurately predict the tails of the response distribution. A new probability model has therefore been introduced to overcome this deficiency. Analysis of simulated response data has demonstrated that this new model, though not perfect, is considerably better than either of the foregoing two models.

Keywords: offshore structures, response, probability distribution, wave loading, Morison's equation.
\end{abstract}

\section{Introduction}

For an offshore structure, wind, wave and gravitational forces are all important sources of loading. The dominant load, however, is normally due to windgenerated random waves. Although some types of these structures can be designed by equivalent deterministic methods, it is inherently much more satisfactory to account for the randomness of the wave loading by establishing the probabilistic properties of the loading and the resulting responses. The major obstacle in the probabilistic analysis of the response due to wave and current 
loading, is the nonlinearity of the drag component of Morison's wave loading [1], which results in non-Gaussian probability distributions for both loading and response [2-5]. The problem is further compounded by current and by intermittent loading on members in the splash zone [6, 7], which have a significant effect on the probabilistic properties of response. Despite these obstacles, it is of crucial importance to develop an accurate model for the probability distribution of response as this information is of great value for efficient probabilistic analysis of an offshore structure. Two probability models have been frequently used for offshore structural response due to random Morison wave loading: a) the Pierson--Holmes distribution [8, 9] and b) a thirdorder polynomial function of a Gaussian random variable [10]. Analysis of simulated data has, however, demonstrated that neither of these two probability models can accurately predict the tails of the response distribution. A new probability model has, therefore, been introduced to overcome this deficiency.

This paper is composed of seven sections. Section 2 is devoted to a brief discussion of the test structure and selected responses. Generation of sample data for the selected responses is discussed in Section 3. The shortcomings of the commonly-used probability distributions in modelling offshore structural response due to Morison wave loading is then studied in Section 4. Section 5 introduces a new probability model for responses. Validation of the new model for both quasi-static and dynamic responses is the subject of study in Section 6 . Finally, the conclusions of this study are presented in Section 7.

\section{Test structure and responses}

The test structure, shown in fig. 1, is in a water depth of $110 \mathrm{~m}$ and is composed of four vertical legs. The diameter of each leg is $1.5 \mathrm{~m}$ with 30 nodal loads representing the distributed load on each leg (total number of nodal loads on the four legs is 120). The drag and inertia coefficients for this structure were taken to be 1.05 and 1.20 , respectively. Waves were assumed to propagate in the positive Y direction.

The following three responses were chosen for investigation: deck displacement, base shear and overturning moment. Both quasi-static and dynamic responses have been calculated for this structure. To achieve different degrees of dynamic response, internal bracings (not shown in the figure) have been introduced between the legs of the structure. Adjustment of the Young's modulus of the bracing elements allows control of the overall stiffness and hence the natural frequency of the structure. The internal bracings were modelled with the following properties: a) no mass, b) stiffness varied by changing Young's modulus, and c) assigned such that no fluid loading is calculated. This was necessary to reduce the computational burden required in the time-domain analysis of the structure.

JCP5 and JCP8 are used to refer to two finite-element (FE) models of the test structure with first mode natural periods of 5.21 and 8.12 seconds (corresponding to $0.192 \mathrm{~Hz}$ and $0.123 \mathrm{~Hz}$ ), respectively. The first ten modes have been used in the evaluation of the dynamic responses for both FE models. Damping 
coefficients (inclusive of hydrodynamic damping) for all modes were assumed to be 0.05 . The effect of added mass has been included in derivation of the dynamic properties of the structure.

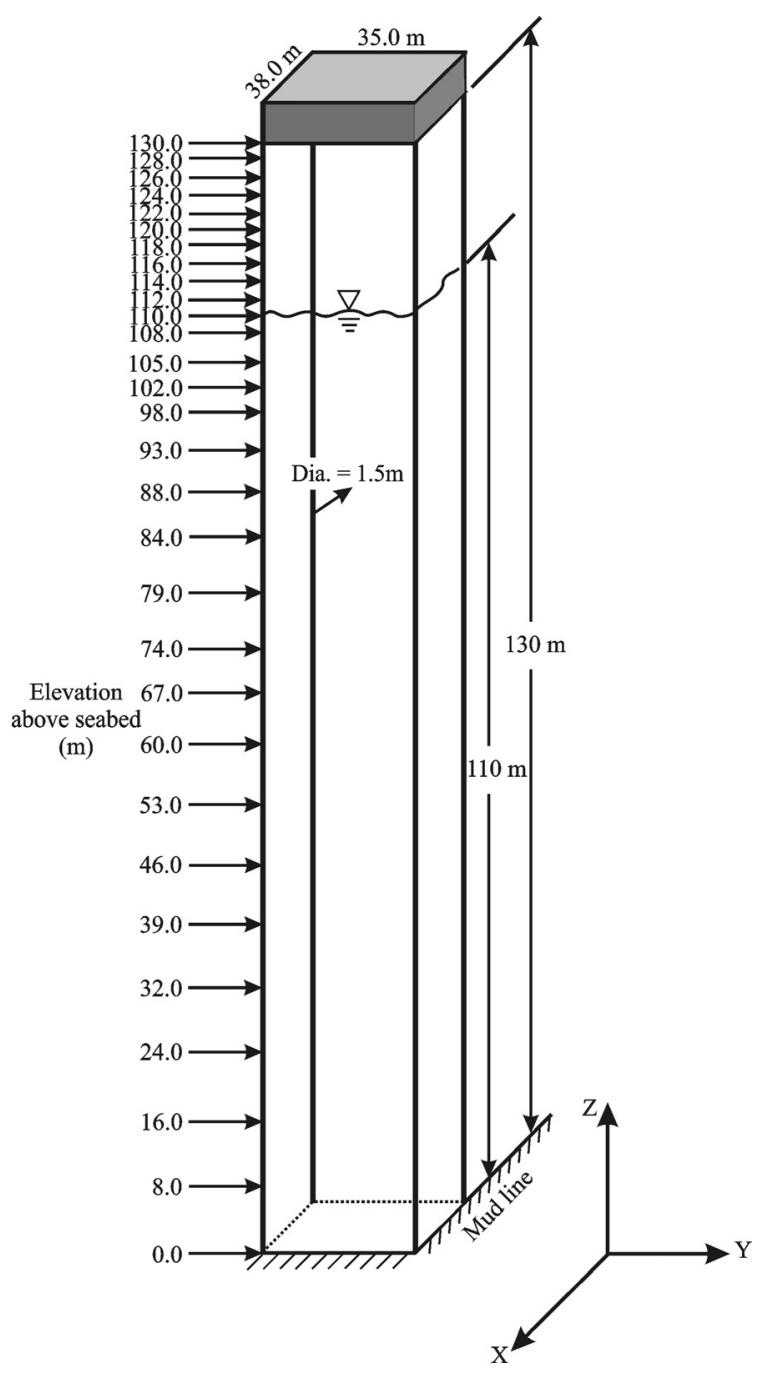

Figure 1: Schematic diagram of the test (JCP5/JCP8) structure.

\section{Generation of sample data}

The foregoing test structure has been subjected to random seas simulated from Pierson-Moskowitz (P-M) frequency spectra. 
In this study, the following definition of the P-M spectrum $[11,12]$ has been used

$$
G_{\eta \eta}(f)=\frac{H_{s}^{2}}{4 \pi T_{z}^{4} f^{5}} \exp \left(-\frac{1}{\pi T_{z}^{4} f^{4}}\right),
$$

where $f$ is the wave frequency in $\mathrm{Hz}, G_{\eta \eta}(f)$ is the surface elevation frequency spectrum $\left(\mathrm{m}^{2} / \mathrm{Hz}\right), H_{s}$ is the significant waveheight in meters and $T_{z}$ is the mean zero-upcrossing period in seconds. Both uni-directional and directional seas have been used in this study. The spreading function for directional seas was taken to be [13]

$$
D(f, \theta)=\frac{\Gamma(1+n / 2)}{\sqrt{\pi} \Gamma(1 / 2+n / 2)} \cdot \cos ^{n}\left(\theta-\theta_{0}\right),
$$

where $\Gamma$ is the gamma function. Waves are assumed to travel in a direction $\theta$ measured from a reference axis (for example, the positive $\mathrm{Y}$ direction of the test structure), and $\theta_{0}$ is the mean (predominant) wave direction. The spreading parameter, $n$, is, in general, a function of frequency; however, for simple analysis, a constant value of $n=4$, independent of wave frequency, is commonly used [14].

Both time simulation $[15,16]$ and principal component simulation (PCS) techniques [17] can be used to simulate long records of response. The PCS technique, which results in independent data points, is considerably more efficient than the time simulation technique (about 25 times); however, it can only be used for simulation of quasi-static responses. Therefore, the more efficient PCS technique has been used to simulate quasi-static responses. On the other hand, dynamic responses have been simulated using the time simulation (TS) technique. For quasi-static responses, the seas were assumed to be directional, while, to reduce the computational effort, the seas for dynamic responses were assumed to be uni-directional. The current speeds for quasi-static responses were assumed to be 0.00 and $\pm 1.50 \mathrm{~m} / \mathrm{s}$, and those for dynamic responses were assumed to be 0.00 and $\pm 0.90 \mathrm{~m} / \mathrm{s}$. $H_{s}$ and $T_{z}$, as measured by an observer moving with the current, were taken to be $\left[H_{s}=15 \mathrm{~m}, T_{z}=13.75\right.$ seconds $]$ and $\left[H_{s}=5 \mathrm{~m}, T_{z}=7.94\right.$ seconds $]$, respectively.

Surface elevation and corresponding water particle kinematics at different nodes were simulated according to Linear Random Wave Theory (LRWT). A common industry practice for evaluation of wave kinematics in the free surface zone consists of using linear wave theory in conjunction with empirical techniques to provide a more realistic representation of near-surface water kinematics. Couch and Conte [18] offer a review of these techniques. While is it known that vertical stretching [19] is not the most accurate, it is representative, does not introduce nonlinearities, and is computationally more efficient; hence it was adopted for this study. 


\section{A review of the existing probability models}

\subsection{The Pierson--Holmes distribution}

The Pierson--Holmes (P-H) distribution [8] was first introduced as a probability model for Morison wave loading on a short segment of a submerged vertical cylinder for a stationary sea state. According to Morison's equation [1], the waveinduced horizontal force per unit length on a vertical submerged cylinder (cylinder diameter / wavelength $<1 / 5$ ) is the sum of a nonlinear drag component and a linear inertial component. The empirical equation can be expressed as

$$
\begin{aligned}
& F=F_{d}+F_{i}=k_{d}(\bar{u}+u)|\bar{u}+u|+k_{i} \dot{u} \\
& k_{d}=C_{d} \rho \frac{D}{2} ; k_{i}=C_{m} \rho \frac{\pi D^{2}}{4},
\end{aligned}
$$

where $F_{d}$ and $F_{i}$ are the drag and inertial components of wave loading; $D$ is the cylinder diameter; $C_{d}$ and $C_{m}$ are empirical drag and inertia coefficients; $\rho$ is the water density; $\bar{u}$ and $u$ are the mean (current) and fluctuating parts of the undisturbed horizontal component of water particle velocity at the centre of the cylinder, respectively. Finally, $\dot{u}$ is the horizontal component of water particle acceleration at the centre of the cylinder.

In its most general form, the Pierson--Holmes distribution is defined as [9]:

$$
\begin{aligned}
& Y=\beta_{0}+\beta_{1}\left(X_{1}+\gamma\right)\left|X_{1}+\gamma\right|+\beta_{2} X_{2} \\
& E\left[X_{1}\right]=E\left[X_{2}\right]=0 ; \quad \sigma_{X_{1}}=\sigma_{X_{2}}=1 ; \quad \rho_{X_{1}, X_{2}}=0,
\end{aligned}
$$

where $Y$ is the random variable with Pierson--Holmes distribution; $\beta_{0}, \beta_{1}, \beta_{2}$ and $\gamma$ are the four parameters of the distribution, and $X_{1}$ and $X_{2}$ are two independent, standardized, and jointly-Gaussian random variables. $E$ is the expectation operator, and finally, $\sigma$ and $\rho$ stand for the standard deviation and correlation coefficient, respectively.

\subsection{The third-order polynomial distribution}

In the case of the third-order polynomial model [10], the probability distribution of response is assumed to be the same as that of the random variable $Y$ defined as a third-order polynomial function of a standardized Gaussian random variable $(X)$. That is,

$$
Y=\alpha_{0}+\alpha_{1} X+\alpha_{2} X^{2}+\alpha_{3} X^{3}
$$

where $\alpha_{0}, \alpha_{1}, \alpha_{2}$ and $\alpha_{3}$ are the four parameters of the distribution. 


\subsection{Reliability of the P-H and the third-order polynomial distributions}

The principal component simulation procedure [17] was used to simulate 524288 data points for both quasi-static base shear and quasi-static overturning moment of the test structure shown in fig. 1. As an example, the probability distributions of the simulated base shear and those of the fitted P-H and third-order polynomial distributions for the case of a negative current are shown in fig. 2 . As observed, both probability distributions fail to accurately model the positive tail of the response distribution. It can, therefore, be concluded that a more accurate probability model for offshore structural response is required.

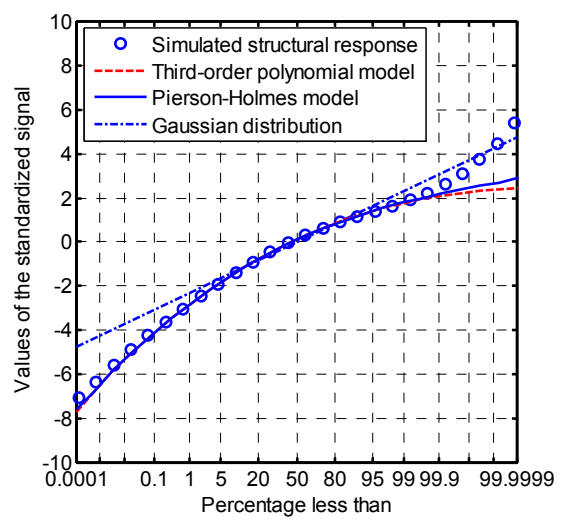

Figure 2: Performance of the $\mathrm{P}-\mathrm{H}$ and the third-order polynomial distributions. Quasi-static base shear, directional seas, current $=-1.5 \mathrm{~m} / \mathrm{s}, H_{s}=15 \mathrm{~m}$.

\section{A new probability model for response}

The following model is proposed for offshore structural response due to Morison wave loading:

$$
Y=\lambda_{0}+\lambda_{1} X+\lambda_{2}(X+\gamma)|X+\gamma|+\lambda_{3}|X|^{3}
$$

where $X$ is a standardized Gaussian random variable, and $\lambda$ 's and $\gamma$ are the five parameters of the probability model. The third and the fourth terms on the right hand side of the foregoing model account for the asymmetry in the response probability distribution due to current, and load intermittency on members in the splash zone, respectively. The second term accounts for the fact that forces on different nodes are not fully correlated and as a result, the response distribution tends to be closer to a Gaussian distribution than that of an individual nodal load. It also accounts for the fact that dynamic responses tend to be closer to Gaussian distribution than quasi-static responses. The methodology for estimation of the parameters of this model will be discussed in a future publication. 


\section{Validation of the new probability model}

In this section the reliability of the new probability model for both quasi-static and dynamic responses is investigated.

\subsection{Quasi-static responses}

The same quasi-static response data as that generated in section 4.3 has been used for investigating the reliability of the new model. As an example, the probability distribution of the simulated quasi-static base shear and that of the new model fitted to the data are shown in fig. 3. It is observed that the agreement between the two is very good. It should, however, be considered that for $H_{s}=15 \mathrm{~m}$, structural responses are strongly drag-dominated. Therefore, these responses have also been calculated for $H_{s}=5 \mathrm{~m}$ to establish whether the new distribution is a good probability model for the response for cases when the inertial component of loading is significant. The results (not shown here) indicate that corresponding probability distributions are in very good agreement. It can therefore be concluded that the new distribution is a reliable probability model for quasi-static responses.

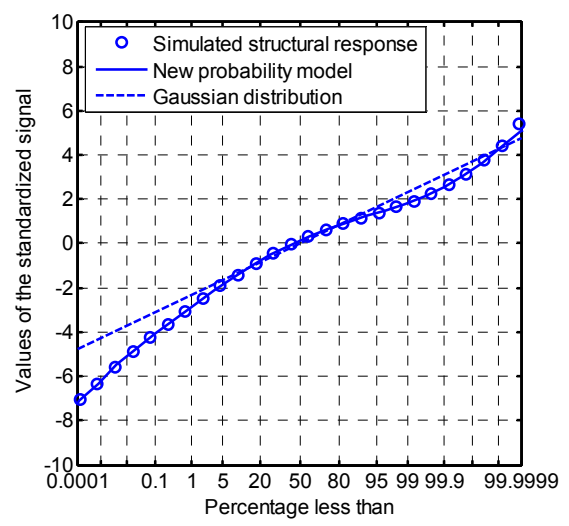

Figure 3: Reliability of the new distribution in modelling quasi-static responses. Base shear, directional seas, current $=-1.5 \mathrm{~m} / \mathrm{s}$, $H_{s}=15 \mathrm{~m}$.

\subsection{Dynamic responses}

Time-domain simulation has been used to simulate dynamic responses of about 64 hours duration. Six responses, i.e., deck displacements, base shears and overturning moments of both JCP5 and JCP8 structures have been investigated. In all cases the agreement between distributions of simulated structural responses and the fitted probability models are good or very good. As an example, the results for dynamic base shear of the JCP8 structure with $H_{s}=15 \mathrm{~m}$ is shown in fig. 4. Furthermore, the frequency spectrum of the response is shown in fig. 5, to 
give an indication of the importance of the dynamic behaviour of the test structure. Similarly, the results (not shown here) indicate that the new distribution is also a good model for dynamic responses for $H_{s}=5 \mathrm{~m}$. It can therefore be concluded that the new distribution is a valid probability model for both dynamic and quasi-static responses.

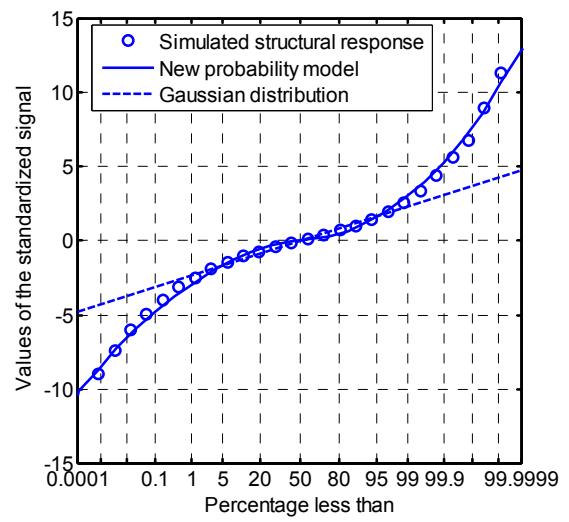

Figure 4: Performance of the new distribution in modelling dynamic response. Base shear, JCP8 structure, uni-directional seas, current $=0 \mathrm{~m} / \mathrm{s}, H_{s}=15 \mathrm{~m}$.

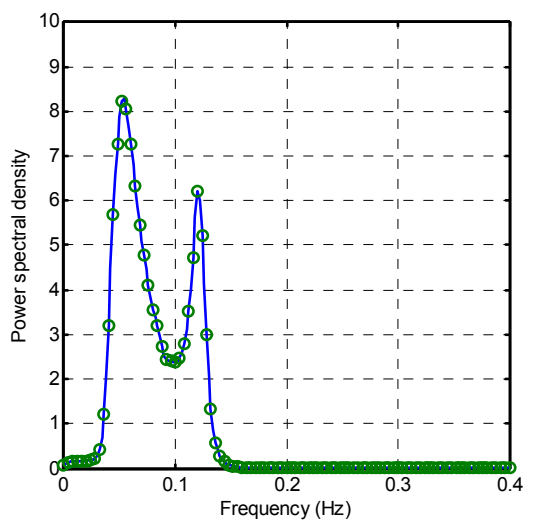

Figure 5: Frequency spectrum of dynamic base shear. JCP8 structure, uni-directional seas, current $=0 \mathrm{~m} / \mathrm{s}, H_{s}=15 \mathrm{~m}$.

\section{Conclusions}

- $\quad$ This paper is concerned with probability distribution of offshore structural response due to Morison wave loading. 
- Analysis of simulated data has shown that neither the Pierson--Holmes nor the third-order polynomial distributions can accurately model the tails of the response distribution.

- A new probability model has been introduced to overcome this deficiency. The distribution has proved to be a successful probability model for both quasi-static and dynamic responses.

- The foregoing conclusion has only been tested for the response of linear structures. However, the model may also prove successful for cases when the relationship between response and nodal loads is not strictly linear.

- Although the new probability model has only been validated for offshore structural response due to Morison wave loading, it may also prove to be a successful model for offshore structural response due to other types of wave loading. This proposition needs to be investigated.

\section{Acknowledgements}

The author wishes to extend his gratitude to the UK Health and Safety Executive for sponsoring elements of this work, and in particular to $\mathrm{Mr}$ Malcolm Birkinshaw, the Project Officer. Thanks are also due to Mr. Mark Manzocchi and Mr Duncan Graham of Atkins Ltd. for their most valued contributions in data provision. Thanks are also due to my colleagues Professor Richard Burrows and Mr. RG Tickell for help in interpretation of the results of this study.

\section{References}

[1] Morison, J.R., O’Brien, M.P., Johnson, J.W., \& Shaaf, S.A., The force exerted by surface waves on piles. AIME Petroleum Transactions, 189, pp. 149-154, 1950.

[2] Borgman, L.E., Random hydrodynamic forces on objects. The Annals of Mathematical Statistics, 38, pp. 37-51, 1967.

[3] Tickell, R.G., Continuous random wave loading on structural members. The Structural Engineer, 55(5), pp. 209-222, 1977.

[4] Burrows, R., Probabilistic description of the response of offshore structures to random wave loading. Mechanics of Wave Induced Forces on Cylinders, ed. TL Shaw, Pitman Advanced Publishing Program: London, pp. 577-595, 1979.

[5] Eatock Taylor, R., \& Rajagopalan, A., Superharmonic resonance effects in drag dominated structures. Proc. of the 2nd Int. Symp. on Integrity of Offshore Structures, Glasgow, Scotland, pp. 85-104, 1981.

[6] Tung, C.C., Effect of free surface fluctuation on total wave force on cylinder. ASCE, J. Engineering Mechanics, 121(2), pp. 274-280. 1995.

[7] Liaw, C.Y., \& Zheng, X.Y., Polynomial approximations of wave loading and superharmonic responses of fixed structures. ASME, J. Offshore Mechanics and Arctic Engineering, 25(3), pp. 161-167, 2003.

[8] Pierson, W.J., \& Holmes, P. Irregular wave forces on a pile. ASCE, J. Waterways and Harbours Division, 91(WW4), pp. 1-10, 1965. 
[9] Najafian, G., Burrows, R., Tickell, R.G., Metcalfe, A.V., \& Hearn, G.E. Pierson--Holmes: A probability distribution for stochastic modelling of wave-induced offshore structural loading and response. Proc. of the 19th Int. Offshore Mechanics and Arctic Engineering Symp., New Orleans, USA, Paper No. OMAE-00-6119, pp. 1-8, 2000.

[10] Winterstein, S.R., Ude, T.C., \& Kleiven, G., Springing and slow-drift responses: predicted extremes and fatigue vs. simulation. Proc. of the 7th Int. Conf. on the Behaviour of Offshore Structures, 3, pp. 1-15, 1994.

[11] Pierson, W.J., \& Moskowitz, L.J. A proposed spectral form for fullydeveloped wind seas based on the similarity theory of S.A. Kitaigorodskii. J. Geophysical Research, 69(24), pp. 5181-5190, 1964.

[12] Tucker, M.J., Waves in ocean engineering: measurement, analysis, interpretation. Ellis Horwood: London, 1991.

[13] Miles, M.D., \& Funke, E.R., A comparison of methods for synthesis of directional seas. ASME, J. Offshore Mechanics and Arctic Engineering, 111, pp. 43-48, 1989.

[14] Barltrop, N.D.P., Floating structures, a guide for design and analysis. Oilfield publications: Herefordshire, England, 1998.

[15] Rodenbusch, G., Random directional wave forces on template offshore platforms. Proc. of the 18th Annual Offshore Technology Conf., Houston, USA, OTC 5098, pp. 24-30, 1986.

[16] Morooka, C.K., \& Yokoo, I.H., Numerical simulation and spectral analysis of irregular sea waves. Int. J. of Offshore and Polar Engineering, 7(3), pp. 189-196, 1997.

[17] Najafian, G., Derivation of statistical properties of wave-induced offshore structural response by principal component technique. J. Ocean Engineering, in press, 2006.

[18] Couch, A.T., \& Conte, J.P., Field verification of linear and nonlinear hybrid wave models for offshore tower response prediction. J. of Offshore Mechanics and Arctic Engineering, 119, pp. 158-165, 1997.

[19] Marshall, P.W., \& Inglis, R.B., Wave kinematics and force coefficients. ASCE Structures Congress, New Orleans, Louisiana, U.S.A, 1986. 